\title{
A New Type of Magnetic Birefringence
}

\author{
$\mathrm{BY}$ \\ S. W. Cunnchalkak. \\ (Received for publication, 25th December 1931.)
}

Abstract.

The paper gives an account of the experiments on magnetic double refraction in aqueous solutions of paramagnetic salts, specially of the rare earths, of which preliminary accounts have appeared in 'Nature,' August 29, 1931, page 363, and Oetober 31, 1931. Cerium, praseodymium, didymium (mixture of neodymium and praseodymium) and erbium salts show in aqueous solutions a negative magnetic birefringence comparable in magnitude with that shown by the organie liquids. Gadolinium nitrate does not show an appreciable birefringence. The birefringence is sensibly proportional to the square of the magnetic field. The author fails to confirm in sign as well as in magnitude the results reported by Elias in 1910 who claims to have observed magnetic birefringence in a solution of erbium nitrate. A solution of ferric chloride made in dilute acid to prevent a formation of colloids also shows a negative birefring. ence.

A provisional attempt is made to apply Langevin's theory of magnetic birefringence to the case of cerium nitrate solution, assuming an optical anisotropy for the $\mathrm{Ce}^{+3}$ ion, the assumption being justifiable in view of Rayleigh and Cabannes' experimental observation that the rare geses, including $\mathrm{X}_{\mathrm{enon}}$ to which the $\mathrm{Ce}^{+3}$ ion is a near approach in strueture, shows a depolarisation of transversely scattered light. The theory with some simplifying assumptions, gives the right order of magnitude for the birefringence.

A parallelism appears to exist between the birefringence and the Faraday effect in these solutions in that those solations which show the 
negative birefringence also show a negative Faraday effect, gadolinium nitrate solution showing neither to an appreciable extent. The negative Faraday effect is, according to Ladenburg's theory, a consequence of the paramagnetic orientation of the ions. It appears that the two phenomena are related to each other.

\section{Introduction.}

Transversal magnetic double refraction which is the magnetic analogue of the electro-optic Kerr effect or electric double refraction, was first successfully demonstrated by Majorana ${ }^{1}$ in 1902 in the case of colloidal solutions of iron. The effect in pure homogeneous liquids, free from colloids was discovered by Cotton and Mouton ${ }^{2}$ later in 1907 in the case of nitrobenzene and other organic aromatic liquids and some aliphatic liquids also; and it is now known that the Cotton-Mouton effect is a general phenomenon exhibited by the organic liquids, both aromatic and aliphatic, to a more or less extent. ${ }^{3}$ Some inorganic groups like nitrate and nitrite ${ }^{4}$ ions are also known to exhibit a feeble magnetic birefringence. All these cases of magnetic birefringence in pure homogeneous liquids were, however, restricted to the diamagnetic class. Experiments were performed in this laboratory towards the end of May and early in June 1931 on aqueous solutions of paramagnetic salts of rare earths. Many of these are known to exhibit a negative Faraday effect which is due to their paramagnetism. Samples of cerium and didymium (a mixture of praseodymium and neodymitim) nitrates locally available showed

1 Q. Majorana : Accad. Lincei Atti., 11, 374 and 463 (1902).

2 Cotton and Mouton: O. R., 148, 870 (1907).

? A list of substances studied up to $\mathbf{A 9 2 9}$ is given in Landolt Bornstein Tebellen. Zweter Erganzungsband, p. 913. Also, 8. W. Chinehalkar, Ind. Jour. Phys., 6, 165 (1931); Scherer, C. R., 192, 1223 (1981).

1 Nitric acid-see Landolt Bornstein Tabellen. Also M. Ramanadham, Ind. Jour. Phys., 5, 15 (1929); Haque, Q. R., 190, 789 (1930) 
that a distinct magnetic double refraction was shown which was far greater than could be accounted for by the nitrate ion and was of the opposite sign; and it was shown by cerium chloride solution also. Cerium was slightly coloured due to an impurity. Pure cerium salts were later found to exhibit the phenomenon, and the existence of the effect was reported by Sir C. V. Raman in the body of an article to the Special Faraday Supplement to 'Nature' of August 29, 1931. The work was further continued when other rare earths were available and a brief note about it was reported in the issue of 'Nature,' dated October 31, 1931. ${ }^{6}$ The object of this paper is to give an account of this work on the magnetic birefringence in solutions of paramagnetic salts.

\section{Experimental.}

The experimental arrangement was the same as was used by the author for measuring the magnetic birefringence in aliphatic liquids. ${ }^{3}$ White light from a 1000 c.p. pointolite lamp was employed. The compensator used to detect and measure the double refraction was the strained glass balfshade elliptic polariser and analyser by Forest Palmer, with the arrangement for applying and varying the pressure as described in that paper. The solution to be experimented upon was kept in a straight glass tube $33 \mathrm{cms}$. long, placed in a corresponding long gap of the special electromagnet. The method of measurement was a comparative one. For lower concentrations of solutions the standard of comparison was acetone and for higher ones carbon disulphide, the latter being compared with nitrobenzene. A current of 11 amps. was passed through the electromagnst giving a field of 24000 gauss when lower concentrations were used. In order that

5 Nature : 128, 363 (1931).

C C. V. Raman and 8. W, Chinchalkar: Nature, 128, 758 (1981), 
too high a load may not be required to be placed on the compensator plate in the case of higher concentration of solutions, a lower current of about 2 amps. was used, so that the compensating load was of the same order of magnitude as in the case of acetone and the lower concentrations.

Cerium nitrate (purest), erbium nitrate, didymium nitrate and ferric chloride (crystals 'for analysis ') were of Kahlbaum's manufacture. Pure gadolinium nitrate and praseodymium oxide were kindly lent by Dr. Pulin Behari Sarkar of the College of Science, Calcutta. Praseodymium chloride was prepared by dissolving the oxide in concentrated hydrochloric acid and diluting the solution to a known strength, a slight excess of the acid remaining in solution. 'Didymium' is known to contain larger portion of neodymium than praseodymium. Erbium nitrate was found by a comparison of the absorption spectrum through different thicknesses of the solution in a wedge-shaped cell with a didymium nitrate solution, to contain about 4 to 5 per cent. of the latter as impurity. Ferric chloride solution was prepared by dissolving the crystals in dilute hydrochloric acid.

\section{Variation with Magnetic Field.}

Before giving the actual results obtained with various substances, a test made about the variation of the magnetic birefringence with field strength will be given. Magnetic birefringence in organic liquids is known to vary as square of the field strength. Two aqueous solutions of cerium nitrate were compared with benzene (Merk's pure) and carbon disulphide (Kahlbaum) using two different field strengths. Resnlts are given in Table $I$. The numbers refer to mean changes of compression of the compensator plate in grams weight required to compensate the birefringence and are proportional to the latter. 
TABLE I.

\begin{tabular}{c|c|c|c|c}
\hline \multicolumn{2}{c|}{ Cerous Nitrate Solutions. } & \multicolumn{2}{|c|}{ B } & Ratio $\frac{\mathrm{A}}{\mathrm{B}}$ \\
\hline 1 & 2 & Benzene & $\mathrm{CS}$ & \\
\hline 285 & & -240 & & $-1 \cdot 19$ \\
460 & & -385 & & $-1 \cdot 20$ \\
& 185 & & 215 & 0.86 \\
& 310 & & 370 & $0 \cdot 84$ \\
\hline
\end{tabular}

It is found that the ratio of the birefringence in the solution to that in the organic liquid is constant within the experimental error when the field strength is varied, showing that in solution it varies in the same way as in the organic liquid, i.e., as the square of the magnetic field.

\section{Results.}

Table II gives the results obtained, on the scale acetone $=+1 \cdot 6$ which corresponds to nitrobenzene $=+100$ after Cotton and Mouton. Values for the two concentrated solutions marked * are given by comparison with nitrobenzene via carbon disulphide at $24^{\circ} \mathrm{C}$. Due to the same law of variation with field strength holding for both the organic liquids and these solutions, the constant of birefringence can be given relative to that of one organic liquid (nitrobenzene) though the strong and the weak solutions are compared with different organic liquids at different field strengths. The Cotton-Mouton constant for organic liquids is known to be defined by

$$
\mathrm{C}=\frac{d}{l} \frac{1}{\lambda \bar{H}^{2}}=\frac{n_{p}-n_{s}}{\lambda \mathrm{H}^{2}}
$$


where $n$, and $n$, are refractive indices of light vibrations parallel and perpendicular to the field and $d$ is the distance retardation between a thickness $l$ of the liquid. Concentrations of praseodymium and cerium chloride solutions are given in terens of equivalent nitrate for comparison with the other nitrates.

TABLE II.

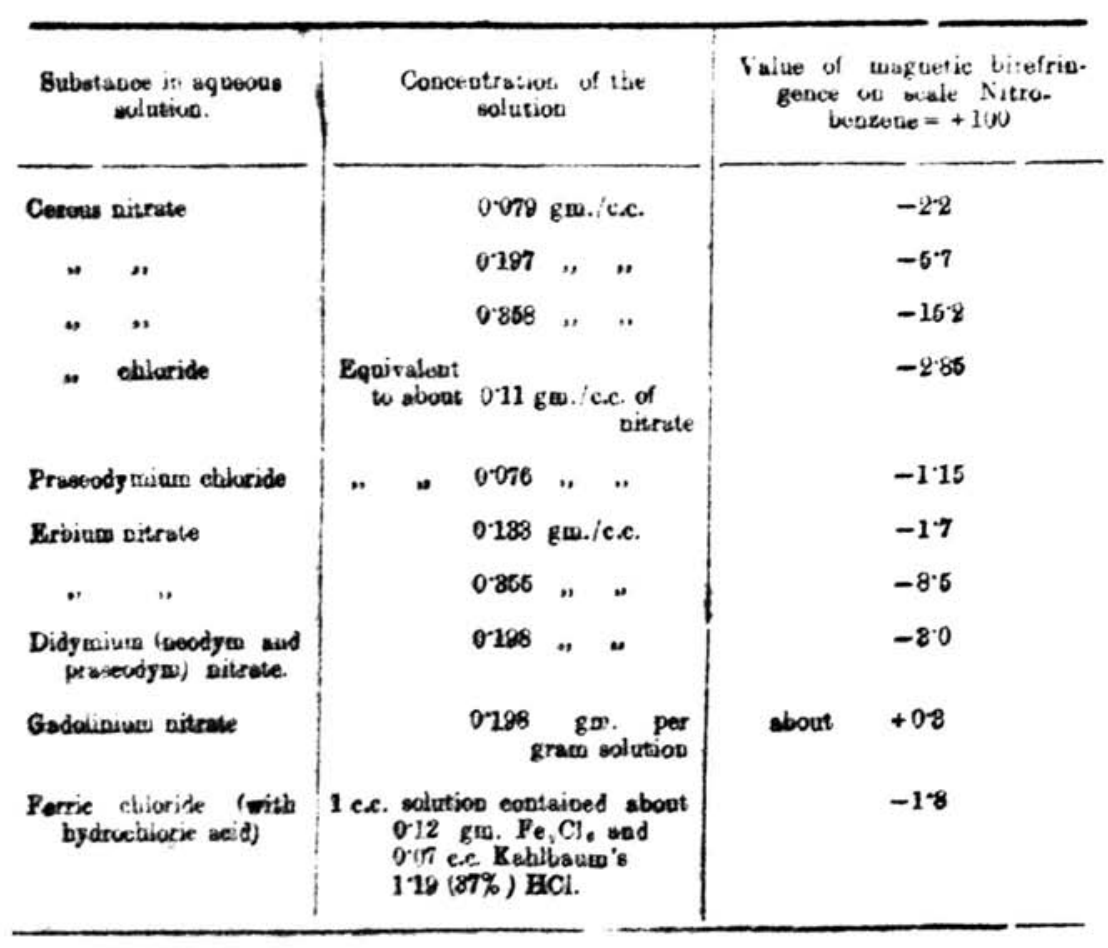

\section{Discussion.}

Table II shows that the effect even in the weak coneptrations is very crsypirusus. No correetion has been made for water and the nitrate ions. It may be remembered that water buws a negative birefringence -0.15 and the vitrate ions in a conventrations corresponding to $0.55 \mathrm{gm}$. 
cerium nitrate per c.c. of solution would give a value of about

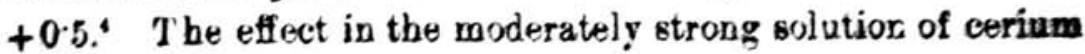
nitrate in Table II is comparable with that of carbon disulphide (-17.9) which is the strongest negatively birefringent organie liquid known, while in a more concentrated solution in Table I, the birefringence is greater than that in benzene in magnitude, and is thus of the same order as that exhibited by the organic liquide of the aromatic class.

Of the different substances, cerium nitrate shows much larger magnetic birefringence than an equiralent solution of any of the others, though it is interesting that the $\mathrm{Ce}^{+3}$ ion has the smallest magnetic moment. The two lower concentrations of cerium nitrate showed proportionality with concentration, which is only approximate, the higher concentration being a little more birefringent than indicated by a strict proportionality. The third concentrated solution, however, shows clearly that the proportionality is not obeyed, the departure baing here more pronounced than in the previous pair. Erbium also shows a similar departure.

Elias ${ }^{7}$ had reported in 1910 to have observed a magnetic birefringence in a solution of erbium nitrate. The concentration of his solution was $0.56 \mathrm{gm} . \mathrm{Er}_{2} \mathrm{O}_{8}$ per c.c. solution, which is equivalent to about $1.04 \mathrm{gm}$. of erbium nitrate per c.e. The value of the birefringence he obtained was about 400 , being positive in sign. The author however finds it to show a negative birefringence, and though the concentration of his olution is about one-third that of Elias, the magnitude of birefringenee is about one-fiftieth. The ebservation of Elias is, therefore, not confirmed, by the author.

Gadolinium nitrate solution is interesting in that though it is strongly paramagnetic, the $\mathrm{Gd}+8$ ion having a magnetic moment of about 8 Bohr magnetcons as compared with $\mathrm{Co}^{+8}=2.5, \mathrm{Pr}^{+8}$ and $\mathrm{Nd}^{+3}$ each 3.5 , and $\mathrm{Er}+8=9.5$ neariy,

1 G. J. Eliwa, Verheod. Deut. Pbys. Gesselischsf., 1610, p. 965.

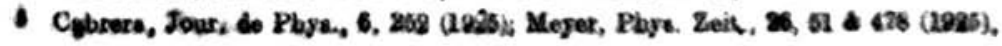


the birefringence observed in it is a very feeble one, of the same order as, though slightly more than, the correction for water and nitrate ions. A rough estimate indicates that the positive birefringence due to the nitrate ions will have a small excess over the negative one due to water though probably less than the value obtained for the solution, showing a possibility of a very feeble positive birefringence for the $\mathrm{Gd}+3$ ion. The point, however, cannot be decided without more precise measurements and corrections. However, as compared with the other rare earth solutions, the effect in gadolinium nitrate, if any, is negligibly small.

A solution of yttrium nitrate of Merck's specimen showed a negative birefringence. The material was slightly coloured and, therefore, impure, and indicated about $1 \%$ of didymium impurity and much more of erbium. Whether all the birefringence observed is due to the impurity bas not yet been ascertained.

A solution of ferric chloride made in the presence of acid is seen to show a negative magnetic birefringence of the same order as that given by the rare earth solutions. It is not likely that colloids would be present in the solution when it is made in dilute acid.

A solution of manganous chloride containing about 0.7 gm of the salt per c.c. solution showed no detectable magnetic birefringence.

\section{The 'Paramagnetic' Birefringence and the 'Paramagnetic' Faraday Effect.}

It is interesting to find that the above solutions of paramagnetic salts which show a negative birefringence also show a negative Faraday effect. ${ }^{2}$ All known substances

- $E, j$ G. J. Elisa, Ann. đ. Phg9., 38, 290 (1911), 
showing a negative Faraday effect with the exception of titanium tetrachloride, are paramagnetic (though the converse is not true) and most of them are salts of rare earths. Ladenburg ${ }^{10}$ has given a theory which takes into account the sffect of paramagnetic orientation on Faraday effect, and finds that under certain circumstances a paramagnetic substance can give a negative Faraday effect, and Darwin " has also shown from a general standpoint the existence of a paramagnetic term in the expression for the Faraday Effect. The theory of Ladenburg has found a further confirmation in the work of Becquerel and de $\mathrm{Haas}^{12}$ and others on magnetic rotation at very low temperatures like that of liquid helium, in certain uniaxial crystals containing paramagnetic rare earths, the paramagnetic effect being enormously greater at these low temperatures than the diamagnetic one by a law corresponding to the Curie law of paramagnetics. Faraday effect in a solution of a gadolinium salt does not seem to have been studied, though in discussing the negative rotation in the crystal Xenotime (Y, Er, Gd, phosphate) Kramers and Becquerel ${ }^{23}$ suggest that if it is assumed that the $\mathbf{S}$ state of the $\mathbf{G d + 3}$ ion is 'decomposed' by the electric field in the crystal, it is possible to explain the paramagnetic rotation in Xenotime as probably due to the $\mathrm{Gd}+3$ ion, at the same time pointing out the difficulties to be encountered in making the above hypothesis. The state of this ion is given by the theory of Hund ${ }^{4}$ in which he has calculated the magnetic moments of rare earth ions starting from spectral and atom-building rules and which has been borne out more or less successfully by comparison with experimental values. The whole paramagnetism

10 Ladenburg, Zeit. f. Phy 9., 34, 898 (1925) ; 46, 168 (1925).

11 c. G. Darwin, Proc. Roy. Soc. A, 112, 314 (1926).

12 Becquerel, de Hass, Kramers and otbers; k. Akad. Amsterdam. Proe. aevers? papers in Vol. $32,1929$.

13 Kràmers and Becqnerel, ibid, $32, \mathrm{~L} N 0$ (1929).

14 Eand : Zeit I. Phys., 33, 855 (1925). 
of the $\mathrm{Gd}+3$ ion is due entirely to the spin of the electrons in the incomplete if shell, the orbital moment contributing nothing to it. All the other paramagnetic rare earth ions have an orbital contribution. The solution of gadolinium nitrate employed by the author, was found to give a Faraday effect of $2^{\circ} 47^{\prime}$ as compared with $2^{\circ} 49 \cdot 5^{\prime}$ due to water while the dilute cerous nitrate solution showed $2^{\circ} 3^{\prime}$ nearly. After due correction for water present in the solution, the rotation due to $\mathrm{Gd}^{+3}$ ion in the solution is seen to be negligibly small as compared with that due to $\mathrm{Ce}^{+3}$ or the other rare earth ions.

\section{Application of Langevin's Theory.}

The theory of Langevin and Born ${ }^{15}$ which has been successful in explaining the electric and magnetic double refraction in pure liquids assumes that the molecules of the liquid are electrically (or magnetically as the case may be) and also optically anisotropic. When the liquid is put in the field, they tend to orientate themselves due to the first anisotropy and this tendency to orientate gives the medium doubly refracting properties due to the optical anisotropy of the molecules. The orientation can take place either due to the induced anisotropy, i.e., the moment induced in the molecule by the static field being different along different directions, or due to a permanent moment existing in the molecule. In the case of Kerr effect, electric double refraction, corresponding to an orientation due to both the causes, can be distinguished by considerations of the effect with the molecules possessing or not possessirg a dipole moment; whereas in the magnetic case only the induced (diamagnetic) magnetic birefringence was known.

If the mechanism of birefringence in the paramagnetic salts of rare earths is the same as in the Cotton-Mouton effect 
in organic liquids, except that the orientation is here due to a permanent magnetic moment, an optical anisotropy for the rare earth ions will have to be postulated. These ions are known to have the same structure as the Xenon atom, except for the extra electrons in the inner $4 f$ shell. The rare gases have been shown by Rayleigh ${ }^{10}$ and Cabannes ${ }^{17}$ to show a depolarisation of light scattered transversely to the beam, so that a definite optical anisotropy for the Xenon core may be taken as indicated by experiment, although it is difficult to explain how it arises. The magnetic birefringence on the Langevin-Born theory is given by

$$
\mathrm{C}=\frac{n_{p}-n_{1}}{\lambda \mathrm{H}^{2}}=\frac{\left(n^{2}-1\right)\left(n^{2}+2\right)}{60 n \lambda} \frac{\left(\theta_{1}+\theta_{2}\right)}{\mathrm{A}+\mathrm{B}+\mathrm{C}}
$$

where $\theta_{1}=\frac{1}{k T}\left\{(A-B)\left(A^{\prime}-B^{\prime}\right)+(B-C)\left(B^{\prime}-C^{\prime}\right)+(C-A)\left(C^{\prime}-A^{\prime}\right)\right\}$

snd $\theta_{2}=\frac{1}{k^{2} \mathrm{~T}^{2}}\left\{\left(\mu_{1}{ }^{2}-\mu_{2}^{2}\right)(\mathrm{A}-\mathrm{B})+\left(\mu_{2}{ }^{2}-\mu_{3}^{2}\right)(\mathrm{B}-\mathrm{C})+\left(\mu_{3}{ }^{2}-\mu_{1}{ }^{2}\right)(\mathrm{C}-\mathrm{A})\right\}$

Here A, B, C are the moments induced by a unit electric force of the incident light wave along the three mutually perpendicular directions of the optical ellipsoid of the particles (molecules in organic liquids) giving rise to birefringence in the medium by their orientation. $\mathbf{A}^{\prime}, \mathbf{B}^{\prime}, \mathbf{C}^{\prime}$ are the induced (i.e., dia) magnetic moments along the same three axes, and $\mu_{1}, \mu_{2}, \mu_{s}$ are the components of the permanent magnetic moment $\mu$ along the same three directions respectively. When the theory is applied to the case of paramagnetic rare earth ions, the diamagnetic anisotropy, determined by $\left(A^{\prime}-B^{\prime}\right)$ etc., is comparatively negligible making $\theta_{1}=0$. Also for

\footnotetext{
16 R. J. 8trutt (Lord Rayleigh) Proc. Roy. Boc., 98, 57 (1920),

17 Oabannes: Ann. Phys., 15, 5 (1921).

Oabannes and Lepape, C. R., 179, 325 (192).
} 
simplicity we shall assume the ion to have all axis of optical symmetry so that $A=B$. This reduces the equation (2) to

$$
C=\frac{\left(n^{2}-1\right)\left(n^{2}+2\right)}{60 n \lambda k^{2} T^{2}}\left\{\frac{(A-C)\left(\mu_{1}^{2}+\mu_{2}^{2}-2 \mu^{2}\right.}{2 A+C}\right\}
$$

The direction of the maximum or minimum optical susceptibility of the ions not being known, the components $\mu_{1}, \mu_{2}, \mu_{3}$ cannot be determined; hence a simplification will be made. If $\mathrm{A}$ is taken to be greater than $\mathrm{C}, 2 \mu_{3}^{2}$ should be greater than $\mu_{1}^{2}+\mu_{2}^{2}$ in order that (3) should give a negative magnetic birefringence as experimentally observed. For simplicity, assume that the direction of the permanent magnetic moment is that of the minimum optical polarisability, making

$$
\mu_{3}=\mu \text { and } \mu_{1}=\mu_{2}=0
$$

This will give the upper limit of the constant $\mathbf{C}$ to be expected from the theory. The depolarisation factor $r$ of the transversely scattered light in gases is known to be connected with A, B, C by the relation

$$
\begin{gathered}
\frac{10 r}{6-7 r}=\frac{(A-B)^{2}+(B-C)^{2}+(C-A)^{2}}{(A+B+C)^{2}} \\
\text { which }=\frac{2(A-C)^{2}}{(2 A+C)^{2}}
\end{gathered}
$$

when $A=B$ according to the assumption made above. From (3), (4) and (5) we get .

$$
\mathrm{C}=\frac{\left(n^{2}-1\right)\left(n^{2}+2\right)}{80 n \lambda k^{2} \mathrm{~T}^{2}} \cdot \mu^{2} \sqrt{\frac{5 r_{0}}{6-7 r}} \quad \ldots
$$

(e) will be applied to the case of cerium nitrate solution. Cerium ion has only one electron in the $4 f$ shell, and hence is nearer to the Xenon cure than any other ion. $\mu$ for $\mathrm{Ce}^{+3}$ ion is about 2.5 Bohr magnetons. $r$ is taken for Xenon, and 
after Cabannes $=0.55 \times 10^{-2} . n$ is the refractive index due to the $\mathrm{Ce}^{+3}$ ions which has been calculated from the measured refractivity of cerous nitrate in solution and data for nitrate ions from Fajans and Joos. ${ }^{18}$ Calculated in this way, with molar refractivity for the $\mathrm{C}_{\theta+3}$ ions in solution as 4.80 , the value of $\mathrm{C}$ comes to $-5 \cdot 68 \times 10^{-13}$, which on the scale acetone $=+1 \cdot 6$, comes to $-22 \cdot 7$, the experimental value being $-15 \cdot 2$. the calculated value is of the right order of magnitude. If we try to apply the theory to other solutions, e.g., of erbium nitrate, we find that due to the refractivity and magnetic moment of the ion being both greater than for cerium, the calculated value should be greater, whereas we find the experimental value to be smaller. Also we cannot explain why the birefringence is negative. The above calculations are given to show the order of magnitude to be expected on the theory.

The parallelism between the magnetic birefringence and Faraday effect in these solutions, however, suggests that there may be a connection between the two. An obvious relation seems to be that both are orientation phenomena. Whether a further connection exists as is indicated by Voigt's ${ }^{12}$ theory of magnetic double refraction in sodium and lithium rapours cannot be decided without knowing the dispersion of this magnetic birefringence, specially near the active absorption band responsible for the Faraday effect. Voigt links up the Faraday effect and the magnetic double refraction with inverse Zeeman splitting in the case of absorption bands and the anomalous dispersion in the vicinity of the bands. It is interesting to note that on Voigt's theory the of magnetie birefringence on the longer wave-length side of the active ultra-violet absorption band is to be negative.

18 Fajans aud Juos : Zeit. f, Phys., 28, I (1924); Geffeken, Zeit. t. Phys. Chem. B, 5 116 (1920).

19 Voigt: Graetz Hanjbach der Electrizilat utd der Magnetismus, IV. 
The author's best thanks are due to Prof. Sir C. V. Raman at whose suggestion the work was taken up, for his keen interest and kind guidance. Thanks are also due to Dr. Pulin Bebari Sarkar for his kindness in giving a loan of pure praseodymium oxide and gadolinium nitrate.

Indian Association por tak Cultivation of Scirnck,

210, Bowbazar Strege, Calcutta.

Note.-After the above work was completed a note by Haenny ${ }^{20}$ on the same subject came to hand. His results for cerium nitrate and gadolinium nitrate confirm the author's earlier observations reported in Nature. Haenny has not given a distinct verdict about erbium nitrate.

20 Haenny : C. R. 193, 931, November 16, 1931. 